\title{
Entre survivance des ONG et mise en mouvement : pratiques et débats des féminismes nicaraguayens à l'heure de la globalisation du genre
}

Between NGO survival and movement setting: practices and debates from the Nicaraguan feminisms in the era of "gender globalization"

Delphine Lacombe

\section{(2) OpenEdition Journals}

\section{Édition électronique}

URL : http://journals.openedition.org/conflits/18175

DOI : $10.4000 /$ conflits. 18175

ISSN : $1777-5345$

\section{Éditeur :}

CCLS - Centre d'études sur les conflits lilberté et sécurité, L'Harmattan

\section{Édition imprimée}

Date de publication : 30 décembre 2011

Pagination : 15-37

ISBN : 978-2-296-55700-0

ISSN : $1157-996 X$

\section{Référence électronique}

Delphine Lacombe, «Entre survivance des ONG et mise en mouvement : pratiques et débats des féminismes nicaraguayens à l'heure de la globalisation du genre », Cultures \& Conflits [En ligne], 83 | Automne 2011, mis en ligne le 04 janvier 2013, consulté le 30 mars 2021. URL : http:// journals.openedition.org/conflits/18175; DOI : https://doi.org/10.4000/conflits.18175 


\section{Entre survivance des ONG et mise en mouvement : pratiques et débats des féminismes nicaraguayens à l'heure de la globalisation du genre}

\section{Delphine LACOMBE}

Delphine Lacombe est doctorante en sociologie à l'EHESS, Paris. Sa thèse porte sur la socio-histoire des luttes contre les violences masculines à l'encontre des femmes an Nicaragua (1979-2006). Elle a également mené des enquêtes sur l'bistoire et les débats de la militance féministe en Amérique Latine. Elle a enseigné la science politique à l'université de la Rochelle et la sociologie politique du genre au cycle ibéroaméricain de l'Institut d'Etudes Politiques de Paris.

$\mathrm{V}$

oilà près de vingt ans que la question de la professionnalisation et de «l'ONGisation» des mouvements de femmes sous l'effet des politiques de développement et des agendas onusiens occupe une place centrale dans les débats des féministes latino-américaines et caribéennes. S'il est peu aisé de réduire leur hétérogénéité à une identité commune, force est pourtant de constater que l'inscription de ce féminisme dans l'idée d'une histoire et d'un sort partagés fait sens. Ainsi, aussi diversifiée - et divisée - soit-elle, sa militance est articulée autour d'enjeux communs.

En témoignent notamment les rencontres féministes continentales qui, organisées tous les deux ans depuis leur inauguration en 1981 à Bogotá, constituent une formidable caisse de résonnance transnationale des débats qui animent les mouvements aux échelles régionales et locales ${ }^{1}$. De nombreuses

1. Voir Sternbach Saporta N., Navarro-Aranguren M., Chuchryk P., Alvarez. E. S., "Feminisms in Latin America: From Bogota to San Bernardo", in Escobar A. et Alvarez. E. S., The Making of Social Movements in Latin America: Identity, Strategy, and Democracy, Boulder, CO, Westview Press, 1992, pp. 205-239. Falquet J., " Panorama du mouvement après la Sixième Rencontre féministe latino-américaine et des Caraibes, novembre 1993 ", Cabiers $d u$ GEDISST, n9-10, 1994, pp. 133-146. Falquet J., « De l’institutionnalisation du féminisme latino-américain et des Caraibes ", Cabiers du GEDISST, n²0, 1998, pp. 131-147. Falquet J., «Un mouvement désorienté, la 8 e Rencontre féministe latino-américaine et des Caraïbes ", Nouvelles Questions Féministes, «Féminismes d'Amérique latine et des Caraïbes », 20, 3, 1999, pp. 5-38. 
controverses ont marqué l'histoire de ces rencontres sur les visées, les stratégies et la composition des actions collectives ${ }^{2}$. Lors de la septième, réalisée au Chili en 1996, d'âpres débats ont été lancés par des militantes se revendiquant de l'autonomie, à propos du nouveau tournant qu'avaient pris les mobilisations féministes dans le contexte de la préparation régionale de la conférence internationale des femmes à Pékin (1995). Si la quête d'autonomie avait signifié jusque-là la distanciation vis-à-vis des mouvements révolutionnaires et des partis politiques dominés par les hommes, elle venait acquérir lors de cette rencontre un sens nouveau : celui de la dénonciation d'une «politique du possible ${ }^{3} »$ parrainée par les institutions internationales et bien souvent relayée par les gouvernements, autour de «perspectives de genre » onusiennes considérées comme tout aussi lénifiantes que récupératrices du savoir et des pratiques militantes des femmes. Les autonomes reprochaient à un certain nombre de féministes d'œuvrer au service de l'ordre dominant par leur relais d'un langage aseptisé et dépolitisé sur la pauvreté et sur les rapports femmeshommes. Elles fustigeaient la façon dont certaines, devenues « expertes en genre ", souvent à la tête de grandes $\mathrm{ONG}$, avaient pu s'arroger la représentation de la militance des femmes auprès d'institutions globales en étant rémunérées grassement dans les réseaux de consultance internationaux. En somme, celles qui, de provenances diverses, avaient dès 1993 commencé à agréger leurs vues pour repenser une utopie féministe, radicale, anti-capitaliste et antiraciste, devenaient au Chili (1996) « les autonomes » par opposition à celles, majoritaires, qu'elles qualifiaient alors $d^{\prime}$ « institutionnelles 4 ».

Parmi les arguments de ce courant de pensée et de ces mobilisations antisystème autoproclamées autonomes ${ }^{5}$, la critique spécifique à l'adresse des ONG fut tout aussi centrale que décapante. Considérées comme des compensatrices bon marché de l'ordre néolibéral, elles affirmaient «Nous voyons les ONG comme les technocrates de la pauvreté de genre. » Elles proposaient

2. La rupture avec les organisations politiques de gauche ou l'appel à la double militance parti/mouvements ont été notamment discutés au cours des années quatre-vingt. La question de l'imbrication des rapports de pouvoir et celle des luttes contre le racisme, l'hétéronormativité et la lesbophobie, a été soulevée avec force à partir des années quatre-vingt-dix.

3. $\mathrm{La}$ «politique du possible " a été revendiquée comme telle par les féministes privilégiant le lobbying auprès d'institutions nationales ou internationales, ou le partenariat avec les agences d'aide au développement. On en trouve par exemple une claire formulation dans l'éditorial de la revue mexicaine Debate Feminista, año 8, vol. 15, avril 1997. On peut y lire l'affirmation suivante : «l'excès du discours utopique limite la possibilité d'aimer ce qui est possible, et nous ne pouvons pas faire de la politique d'une manière humaine sans adhésion au possible ou sans recherche du possible », cité par Gargallo F., dans « Féminismes multiples : pratiques et idées féministes en Amérique Latine » in Verschuur C. (ed.), «Genre, postcolonialisme et diversité des mouvements de femmes», Cabiers Genre et Développement, n 7 , pp. 277-295, Paris, L'Harmattan. La traduction est de Saskia Velásquez.

4. Voir : Curiel O., «El 7 mo encuentro lésbico feminista : trascendente e histórico », Bogota, 14 février 2007, en ligne : http://www.feministastramando.cl/ index.php?option $=$ com_content\&task $=v i e w \& i d=444 \&$ Itemid $=62$

5. L'histoire complexe du mouvement autonome, dont nous décrivons ici les traits principaux, a été rendue visible et analysée en France grâce aux travaux de Jules Falquet. Nous reprenons avec son autorisation et nos remerciements les citations que J. Falquet a sélectionnées pour un article à paraitre dans Recherches Féministes, "Pour un autre imaginaire politique : vingt 
alors implicitement d'autres formes d'alliances et de redistribution de financements : "réorienter le financement externe dans les réseaux de solidarité vers les mouvements de femmes, sans la médiation des ONG ni des gouvernements, [...] Investir dans la récupération de la terre, le développement de la production alimentaire et la médecine naturelle. Articuler la lutte internationaliste des femmes autour des mouvements et non des centres de pouvoir comme l'ONU ». Les propos sur la coopération étaient tout aussi acerbes : " la coopération a pour objectif de faire circuler l'excédent matériel et humain d'une manière "rationnelle" qui n'affecte pas l'ordre actuel des choses. C'est ainsi que fonctionnent les agences de coopération des gouvernements, fondamentalement : ils nous donnent leurs miettes, et encore avec des conditions $(\ldots) »$.

En jetant ainsi le pavé dans la mare et en osant la confrontation directe avec les «institutionnelles 6 », les « autonomes » ont eu le mérite d'amorcer et de baliser une réflexion critique qui reste d'une brûlante actualité dans la militance comme dans les milieux académiques. Elles ont rendu visible, outre le foisonnement d'ONG sous l'effet de la manne financière de bailleurs de fonds internationaux, l'extension des logiques marchandes et managériales aux activités associatives. Elles ont souligné les glissements de langage consistant à lisser et individualiser les discours féministes : l'émancipation collective des femmes a été supplantée par les stratégies d'empowerment individuel, tandis que la notion de genre est devenue un terme fourre-tout et non conflictuel permettant d'évoquer conjointement les femmes, les relations entre les hommes et les femmes et les identités sexuelles. C'est enfin le rôle instrumental que pouvait avoir désormais l'action publique de genre pour donner gage de progressisme à toute sorte d'institutions ou d'activités qui était montré du doigt ${ }^{7}$.

Outre les nuances apportées par certaines activistes sur les liens entretenus entre institutions et féminismes ${ }^{8}$, la notion même d'autonomie s'est par la

ans de réflexions et de pratiques "féministes autonomes" latino-américaines et caribéennes " issues d'un texte de Mujeres Creando intitulé «Dignité et Autonomie » (1994, pp. 46-51). Pour un travail collectif sur les « féminismes dissidents » voir Curiel O., Falquet J., Masson S. (ed.), "Féminismes dissidents en Amérique Latine et aux Caraïbes », Nouvelles Questions Féministes, 24, 2, 2005.

6. Les institutionnelles étant celles qui ont opté pour l'institutionnalisation de l'action collective, à savoir pour un «processus d'accès aux ressources (symboliques, matérielles, politiques) [...] Les organisations féministes institutionnalisées pourraient ainsi être définies comme celles qui existent grâce au financement des institutions publiques nationales ou internationales ou qui cherchent à en bénéficier, et celles qui sont engagées dans une interaction avec l'État [...] et les organismes internationaux ». Stoffel S., « L'institutionnalisation au service de l'autonomie des féministes chiliennes ", Recherches Féministes, vol.20, n², 2007, pp. 37-59.

7. Pour une réflexion critique sur les institutions internationales et la mondialisation du point de vue du genre, voir Falquet J., De gré ou de force, Les femmes dans la mondialisation, Paris, La Dispute, le Genre du Monde, 2008.

8. On se réfèrera aux nuances apportées par Amalia Fischer dans le numéro de Nouvelles Questions Féministes sur les féminismes dissidents : Fischer A., « Les chemins complexes de l'autonomie », Nouvelles Questions Féministes, op. cit., pp. 65-85. 
suite élargie à d'autres lectures critiques de la globalisation. Le rejet de l'économie néolibérale et capitaliste, la dénonciation de l'hétéronormativité et de la domination post-coloniale ont articulé de nouvelles lectures attentives à l'imbrication des systèmes d'oppression (de race, de classe et de sexe) et ont été le support de nouvelles expressions de radicalité autour des mobilisations des féministes lesbiennes, afrodescendantes, éco-féministes ou encore indiennes ${ }^{9}$.

L'approfondissement critique et la diversification de ces luttes issues du courant autonome continuent néanmoins de constituer la collusion entre féminismes et institutions en vecteur principal de dé-radicalisation des actions collectives. C'est sur ce point que nous souhaitons ici mettre l'accent. Car au moins trois ressorts critiques occultent les configurations plus amples de la militance féminine et féministe. D'abord, les autonomes ont eu vite fait de renvoyer toutes celles qui ne partageaient pas nécessairement leurs vues à une mouvance homogène solidaire et implicitement articulée autour d'objectifs et de pratiques militantes semblables. Ensuite, la dénonciation des effets de structure apolitique des institutions internationales ou de la coopération a caché la manière dont les ressources financières diffusées par ces dernières ont été captées et appropriées par les associations se revendiquant du féminisme. Enfin, nombre d'analyses portant sur les tendances lourdes des actions collectives sous les effets de structure de la globalisation néolibérale ne peuvent araser les trajectoires locales de la militance, ses débats et ses stratégies liés aux contextes politiques nationaux.

C'est à l'appui d'une enquête menée au Nicaragua 10 sur les recompositions de l'action collective féministe post-sandiniste (1990 - 2010) que nous souhaitons montrer, à distance d'un angle de vue dichotomique du type autonomes/institutionnelles, comment les activistes œuvrent dans un continuum fragile entre adaptabilités stratégiques aux ressources et contraintes externes, et politiques de résistances féministes aux gouvernements hostiles à la cause des femmes. Fragile, car non sans tensions entre aspiration voulue par les militantes de «faire mouvement » de la manière la plus indépendante possible des institutions nationales et internationales, et volonté de pérenniser des ONG qui ont largement contribué à façonner les mobilisations. En relocalisant et recontextualisant les débats et les pratiques portant sur la professionnalisation et « l'ONGisation » des associations de femmes, il s'agit de réinscrire les débats intra-militants, l'historicité et la multiplicité des pratiques, le multipositionnement des activistes, au creux d'enjeux politiques localisés qui imposent de resserrer quelque peu l'échelle d'observation.

9. Voir l'introduction de Falquet, Curiel et Masson dans Nouvelles Questions Féministes, op. cit.

10. Ce travail repose sur de nombreux entretiens, recueil d'archives, observations participantes, dans le cadre d'une enquête de doctorat menée depuis 2004 sur la socio-histoire des luttes contre les violences masculines faites aux femmes au Nicaragua - Côte Pacifique (1979-2006). 
Nous analyserons dans un premier temps l'histoire du déplacement de la notion d'autonomie. Elle a d'abord signifié la distanciation vis-à-vis du parti révolutionnaire au sortir des années quatre-vingt en donnant forme à un féminisme de la seconde vague, articulé autour du slogan « le personnel est politique ». Elle a ensuite correspondu à l'appel d'une articulation d'une militance féministe de plus en plus professionnalisée dans les ONG, autour de revendications et de projets indépendants des agendas institutionnels internationaux et nationaux. Nous montrerons comment la quête de construction de mobilisations féministes articulées et capables d'intégrer des revendications multiples (la mise en mouvement) pour éviter les risques d'isolement et de fragmentation des groupes provoqués par l'ONGisation, a fait l'objet de débats et de tensions au sein de la militance. Dans le prolongement de cette analyse, nous prendrons l'exemple de la lutte contre les violences faites aux femmes, pour montrer que l'institutionnalisation et la professionnalisation n'ont pas toujours été synonymes de dé-radicalisation. Nous soulignerons enfin le fait que la question des partenariats internationaux intervient surtout en contrepoint des obstacles nationaux à la prise en compte des revendications féministes. Dans ce contexte, la recherche d'autonomie signifie d'abord être en capacité de construire des marges de manœuvre et de desserrer des contraintes tant nationales qu'internationales.

\section{Affranchissement vis-à-vis du parti révolutionnaire et construction d'un féminisme de la seconde vague}

Issues des luttes révolutionnaires qui ont chassé du pouvoir le dernier représentant de la dictature des Somoza en 1979, puis des organisations féminines subordonnées au Front sandiniste de libération nationale (FSLN ou Front), nombre de militantes sandinistes ont construit leur féminisme dans un rapport de collusion et de collision avec les organisations révolutionnaires à dominance masculine. Supportrices et actrices engagées du régime sandiniste d'inspiration marxiste et d'affirmation nationaliste (1979-1990), guérillères pour certaines d'entre d'elles, elles se sont saisies, au cours des années quatrevingt, de la mise à l'honneur de la rhétorique égalitaire du FSLN pour faire valoir « les droits spécifiques des femmes ». Plusieurs thèmes ont émergé progressivement au nom de la révolution. On peut citer à cet égard le thème du respect de l'intégrité physique et psychologique des femmes, du droit de dénoncer les hommes violents dans tous les domaines de la vie (privée, publique, partisane et professionnelle), de l'autonomie corporelle et sexuelle, ou encore du droit à la contraception et à l'avortement. Ce faisant, elles osaient défier à plusieurs titres certains supports idéologiques du régime sandiniste et les instances dirigeantes de leur Parti.

Première de leur hétérodoxie, elles soulevaient, en pleine guerre civile entre Armée populaire sandiniste et Contras financés par les États-Unis, des questions jugées secondaires, individualistes et dignes d'un réflexe " petit- 
bourgeois », face à la priorité de défense des intérêts nationaux. En avançant la nécessité de rendre visibles les violences conjugales et sexuelles s'exerçant contre les femmes, elles commençaient à montrer combien celles-ci n'étaient pas moins politiques que la guerre entre ennemis idéologiques. Deuxième hétérodoxie, en indiquant, contre toute consigne du régime, que les rapports entre les femmes et les hommes devaient aussi être lus au filtre de l'antagonisme, et non de la «complémentarité » (aux hommes le travail des armes, aux femmes le travail reproductif de la relève révolutionnaire), elles désindexaient le sort des femmes de celui de l'émancipation nationale, mettaient sur un pied d'égalité les luttes contre le patriarcat et la lutte des classes et affirmaient, en somme, que l'ennemi de classe des femmes avait aussi un sexe, fût-il sandiniste ou contre-révolutionnaire. Troisième hérésie implicite, elles redéfinissaient le domaine du politique, en affirmant que si être bonne révolutionnaire consistait à sacrifier les jouissances personnelles au profit de l'engagement national et partisan, alors la révolution se devait aussi de " pénétrer le royaume des hommes 11 ", celui de la sphère privée et d'en redéfinir le contrat sexuel. Quatrième hétérodoxie, elles entendaient soit réformer l'organisation de masse féminine AMNLAE (Asociación de Mujeres Luisa Amanda Espinoza) pour qu'elle ne soit plus seulement la courroie de transmission des consignes du Parti, soit s'en affranchir et ce afin de mobiliser les femmes autour de leurs «intérêts pratiques et stratégiques 12 ».

Ne trouvant dans cette organisation ni de place ni de temps pour constituer des mobilisations autres que celles censées venir en aide aux hommes engagés dans la guerre, nombre de militantes ont utilisé des espaces liés au Parti ou en ont créé de nouveaux pour dessiner des lignes revendicatives. Les "Secretarías de la Mujer» de différents syndicats tels que CONAPRO H-M (Confederación Nacional de Profesionales Héroes y Mártires) ou l'ATC (Asociación de Trabajadores del Campo) ont réuni les femmes selon leur secteur professionnel (services, agriculture). Et c'est par une prise de parole au sein de ces syndicats qu'elles ont réussi à légitimer le droit d'accès à la contraception, ou encore la nécessité de dispositifs les soutenant dans leurs responsabilités familiales. Certains services sociaux (tels que les garderies d'enfants) ont été réclamés devant la pression que représentait un travail agricole et industriel toujours plus lourd en raison des départs de la main d'œuvre masculine à la guerre ou dans les pays voisins ${ }^{13}$. À partir de 1986, des groupes de femmes sandinistes se revendiquant du féminisme se sont organisés de

11. Commentaire de Sofía Montenegro, citation extraite des débats de préparation à la rédaction de la Constitution de 1987. Morgan M., "Founding Mothers, Women's Voices and Stories in the 1987 Nicaraguan Constitution”, Boston University Law Review, 70(1), 1990, p. 38.

12. L'expression est empruntée à Maxine Molyneux. Molyneux M., "Mobilization without Emancipation? Women's Interests, The State and Revolution", in Fagan R., Deere C. D., Coraggio J. L. (eds.), Transition and Development: Problems of Third World Socialism, Berkeley, Monthly Review Press and Center for Study of the Americas, 1986, pp. 280-302.

13. Voir Murguialday C., Nicaragua Revolución feminismo (1977-1990), Editorial Revolución. Madrid, 1990. 
manière indépendante des syndicats et du Front : les collectifs des villes de Matagalpa, de Masaya et de Managua ont inauguré une nouvelle manière de mener la militance en mettant l'accent sur l'aide aux femmes en situation de divorce, victimes de violences masculines (ou de « maltraitances » selon la terminologie de l'époque). Au même moment a été créée à Managua une "officine légale de la femme » proposant des services de conseils juridiques (séparations, pensions alimentaires, violences conjugales). L'expérience sera reproduite dans les antennes locales d'AMNLAE dans la région pacifique. Une autre association prestataire de services (conseils juridiques et accès aux soins), Ixchen, est créée dès 1988. Enfin, prolégomènes d'une militance plus radicale qui entendait procéder à une sorte d'entrisme féministe auprès des plus hautes instances du FSLN, le Parti de la gauche érotique (Partido de la Izquierda Erótica, PIE) réunissait régulièrement et de manière informelle, à la fin des années quatre-vingt, une quinzaine de femmes diplômées des secteurs urbains, toutes proches du pouvoir révolutionnaire ${ }^{14}$.

Ces activités n’ont pas correspondu à la fin des allégeances idéologiques et partisanes. Elles ont toutefois constitué une rébellion significative, soldée souvent par des sanctions politiques. Cela étant, les militantes entendaient aussi affirmer la compatibilité des luttes socialistes et de genre, de telle sorte qu'elles ont toujours soutenu le projet révolutionnaire en espérant qu'il s'approfondisse par l'inclusion des visées féministes. Cela explique aussi le fait que les activités distanciées des assignations partisanes ont aussi reçu les financements de donations étrangères pro-sandinistes et de groupes de solidarités internationaux, le plus souvent centralisées et redistribuées par le Parti-État ou par la fondation qui lui était subordonnée, la Fundación Augusto César Sandino.

Reste que l'affranchissement organisationnel est apparu rédhibitoire à tout un pan de la militance se revendiquant du féminisme. Cette libération progressive des rangs du FSLN s'est doublée d'une transposition aux nouveaux espaces associatifs des femmes d'une expérience organisationnelle solide et des acquis de la discipline révolutionnaire. C'est la co-existence du déplacement et du questionnement de tous ces savoir-faire, associés à la quête d'une autonomisation vis-à-vis du Parti, et plus prosaïquement de la recherche de repositionnements professionnels qui, au sortir des années quatre-vingt, contribueront à drainer nombre de militantes sandinistes et féministes vers des associations indépendantes, à la recherche de financements propres.

14. Sur ces aspects de l'histoire des mobilisations de femmes nicaraguayennes au cours des années quatre-vingt, voir Isbester K., Still Fighting, The Nicaraguan Women's Movement, 19772000, Pittsburgh, University of Pittsburgh Press, 2000. Molyneux M., "Family Reform in socialist states: the hidden agendas”, Feminist Review, 21, 1985, pp. 47-66. Randall M., Las bijas de Sandino, una historia abierta, Managua, Nicaragua, Ediciones Centroamericanas, 1999. Collinson, H. (ed.) Women and Revolution in Nicaragua, New Jersey, Zed books, 1990. 


\section{Foisonnement des ONG et appel à une mise en mouvement : repositionnements professionnels et déplacements de savoir-faire}

Le 25 février 1990 marque alors un tournant politique et social. Daniel Ortega, leader du FSLN et président depuis 1984, perd les élections face à Violeta Chamorro, veuve de Pedro Joaquín Chamorro (journaliste martyr anti-somoziste) et chef de file la coalition anti-sandiniste Unión Nacional de Oposición. Trois ans après les accords de paix, cette date charnière inaugure de nouvelles sociabilités marquées par l'autorisation d'une altérité au FSLN et l'amorce du pluralisme, la pacification et la démilitarisation progressive du pays ${ }^{15}$, ainsi que, bien que théorique mais faisant désormais sens, le déploiement d'une société civile ${ }^{16}$. Décontenancées par une victoire qu'elles n'attendaient pas, les sandinistes se sont senties orphelines d'une révolution, et ce d'autant plus que celle-ci se solda par un bilan humain pour le moins dramatique après des années de guerre. En sus de ce sentiment d'échec et de la crainte de voir les vues les plus conservatrices (et néolibérales) régner sur le pays et défaire quelques avancées législatives du régime sandiniste, le démantèlement des institutions du Parti-État a signifié pour beaucoup la perte d'un emploi comme cadre du Parti ou comme fonctionnaire ${ }^{17}$.

Ce contexte d'instauration de la démocratie et d'ouverture de l'espace civil entraîne alors deux effets majeurs sur la militance des femmes : la liberté de s'organiser autrement, et la possibilité d'être rémunérées par un secteur associatif en construction que financent les ONG internationales et la coopération bi et multilatérale.

\section{Autonomisation vis-à-vis du Parti et "ONGisation »}

Ce qui change, à cette époque, est moins l'afflux de fonds étrangers (qui ont été extrêmement importants tout au long de la décennie sandiniste avec un apport à hauteur de sept cents millions de dollars par an ${ }^{18}$ ), ou même la présence d'ONG internationales, que la possibilité de capter des ressources externes sans médiation partisane. Désormais, la « loi 147 », votée par le parlement en 1992, donne un cadre légal aux associations à but non lucratif, leur permettant d'obtenir leur personnalité juridique non plus auprès du PartiÉtat, mais auprès du bureau de l'Assemblée nationale ${ }^{19}$. Nombreuses sont les

15. 75000 membres de l'Armée Populaire Sandiniste et 22000 soldats de la « Résistance nationale » sont démobilisés au début des années quatre-vingt-dix. Serra V., Héctor L., La Sociedad Civil Nicaragüense, sus organizaciones y sus relaciones con el Estado, Centro de Análisis Socio-Cultural, Universidad Centroamericana, 2007, p. 45.

16. Bataillon G., « Nicaragua : La présidence Chamorro, L’instauration d'un régime démocratique désenchanté ", Problèmes d'Amérique Latine, n 30, juillet-sept 1998, pp. 71-92.

17. 29125 fonctionnaires perdent leur emploi entre 1990 et 1992 (Serra V., op. cit., p. 45).

18. Il s'agit de l'aide officielle au développement, qui ne comprend pas ici l'aide privée. Varela $\mathrm{BH}$ 1998, "Las ONG en Nicaragua : limitaciones y tendencias en su relación con la cooperación internacional” Managua FONG-INIES-CON-SNV cité dans Serra Vásquez, ibid., p. 44. 
ONG internationales qui, déjà actives durant la décennie sandiniste, viendront financer toutes ces initiatives. Un « répertoire des ONG » daté de 200420 en inventoriait soixante-dix, d' « Action contre la Faim - Espagne » à "Werkhof - Allemagne », en passant par les fondations Ebert, Adenauer, Ford, et les ONG Oxfam, Care, Terre des Hommes, Forum Syd, un grand nombre d'entre elles indiquant parmi leurs axes prioritaires de travail : «perspectiva de género » (perspective de genre). Outre le secteur de l'aide privée au développement, les coopérants bilatéraux «solidaires de la révolution » dans les années quatre-vingt, ont continué à investir d'importantes ressources dans le budget national 21 et dans la société civile, à l'aune cette fois de l'appui au «ferment démocratique » que représentent les ONG. Si tous ces acteurs intervenaient pour parer les effets du blocus économique étatsunien dans les années quatre-vingt, ils compensent partiellement et indirectement à partir des années quatre-vingt-dix les conséquences du retrait de l'État amorcé dès la mise en place des mesures d'ajustement structurel en 1987. À travers cette reconduction de fonds privés et publics d'aide au développement captés par les ONG nicaraguayennes, plusieurs milliers d'emplois sont créés. On estimait par exemple qu'au cours de l'année 2004 le secteur associatif avait créé près de huit mille emplois et mobilisé près de cent soixante-dix millions de dollars 22 .

Dans ce contexte, certaines anciennes cadres ou responsables politiques du Parti fondent leurs propres ONG et recyclent leurs compétences au service de projets entamés ou inaboutis pendant la période sandiniste. À titre d'exemple, les membres du Centre des Droits Constitutionnels - Carlos Nuñez Téllez, fondé en 1990 et dirigé par une ancienne juriste assesseure à l'Assemblée nationale du régime sandiniste, leader de l'un des Secretarías de la Mujer en marge du FSLN, rédigent et réussissent à faire voter par les députés, dès 1992, une loi de pénalisation des violences sexuelles. L'ONG propose un programme de formation et de mise en réseaux de « défenseuses populaires » qui enseignent à d'autres femmes le corpus juridique en matière de genre, lesquelles reproduiront à leur tour l'expérience pédagogique dans leurs communautés. Cela n'est pas sans rappeler la manière dont a été menée par le FSLN,

19. C'est en obtenant sa personnalité juridique que l'association à but non lucratif acquiert le statut d'ONG et peut gérer des financements externes, notamment en pouvant ouvrir un compte en banque. Toutes les occurrences ONG dans ce texte correspondent à cette définition. Certains « mouvements » mentionnés au fil du texte n'ont pas choisi d'avoir ce statut justement pour éviter la confusion entre « mouvement social » et « organisation non gouvernementale ». Il est toutefois fréquent que des ONG prêtent leur personnalité juridique à une autre organisation qui n'en a pas, pour que cette dernière puisse exécuter des projets.

20. CAPRI, 2004, Centro de Apoyo a Programas y Proyectos, Directorio ONG de Nicaragua.

21. L'aide officielle au développement a représenté entre 1994 et 2003 en moyenne annuellement 671 millions de dollars, soit près d'un quart du PIB national. Gosparini et alii, Evaluación conjunta del apoyo presupuestario general 1994-2004, Burkina Faso, Malawi, Mozambique, Nicaragua, Ruanda, Uganda, Vietnam, Informe de País Nicaragua, mayo 2006, International Development Department School of Public Policy University of Birmingham.

22. Serra V., op. cit., p. 84. 
au début des années quatre-vingt, la campagne d'alphabétisation à destination de la population nicaraguayenne majoritairement illettrée. Les pratiques sont ainsi composites entre rhétorique émancipatoire sandiniste et méthodes du régime révolutionnaire et langage nouveau du «droit d'avoir des droits ». La mise à l'honneur internationale des «droits humains » par les conférences onusiennes ainsi que la préparation de la conférence de Pékin auxquelles prennent part à différentes échelles des leaders féministes insufflent un nouveau discours qui innerve peu à peu les expériences revendicatives. Certaines associations deviennent prestataires de services et offrent aux femmes un accès aux soins à l'image de l'organisation SI MUJER (Servicios Integrales para la Mujer) tout en s'engageant dans la lutte pour les droits sexuels et reproductifs. D'autres, avec à leur tête d'anciennes cadres de l'organisation de masse féminine, font des luttes contre les violences masculines leur priorité, comme à Managua (Coletivo 8 de marzo) ou à Estelí (Acción $\mathrm{Ya}$ ); elles accompagnent les femmes victimes de violences et commencent à mener des campagnes d'information à destination des institutions et des habitants de leurs villes ou quartiers. Une fondation telle que Puntos de Encuentro, à l'initiative d'une Française et d'une Étatsunienne issues de la mouvance internationaliste solidaire de la révolution, entame un travail de divulgation des analyses féministes. Elles créent La Boletina, une revue mensuelle dont le tirage à 26000 exemplaires la place dans les publications les plus diffusées du pays. La fondation produit «Sexto Sentido », un feuilleton télévisé à destination de la jeunesse, qui aborde sans fard des thèmes tels que la sexualité, les violences conjugales et sexuelles. Ce nombre de plus en plus important d'organisations indépendantes du FSLN coexiste avec les représentantes locales d'AMNLAE toujours supportrices du parti sandiniste, ainsi qu'avec de plus en plus d'associations axées sur l'indépendance économique des femmes dans les zones rurales (micro-crédits autogérés, propriété de terres ou de bétail), telles que Xochitl Acatl à Malpaisillo, ou avec des associations mixtes communautaires où «le genre » interviendra progressivement comme une perspective incontournable de travail.

Une enquête conduite par quelques militantes féministes sur les transformations de « leur » mouvement entre 1990 et 1995 montre ainsi qu'il était possible de recenser en 1997 près de deux cent cinquante organisations, groupes, ou collectifs consacrés à la cause des femmes, toutes activités et statuts confondus (investigations, prestations de services aux femmes, petites associations naissantes, axes spécifiques de travail au sein d'ONG déjà constituées, médias). À côté de celles-ci, elles listaient vingt fondations ou institutions privées et trente et une institutions nationales publiques travaillant à l'adresse des femmes ${ }^{23}$. Pour beaucoup construites par la génération des féministes sandinistes, les ONG très hétérogènes résultent le plus souvent d'une expérience

23. Montenegro S. et alii., Movimiento de mujeres en Centroamérica, Managua, Centro Editorial de la Mujer (CEM), 1997. 
déjà acquise d'extraversion vers les ressources étrangères et sont le lieu, dès le début des années quatre-vingt-dix, de pratiques hybrides ${ }^{24}$ entre prestations de service et travail féministe de transformation des rapports sociaux de sexe.

\section{"Faire mouvement»}

Dès 1992 se pose la question de l'articulation organisationnelle de toutes ces initiatives diverses et polycentriques, pour rassembler les militantes à distance de l'organisation de masse féminine AMNLAE.

Les premiers débats émergent sur les risques de dépolitisation et de fragmentation d'une action collective longtemps structurée par le Parti. Après l'échec de la réforme d'AMNLAE afin que cette organisation constitue un front unitaire féministe indépendant, un rassemblement est organisé à la manière d'un véritable «Que faire ? » relatif au projet féministe. La rencontre «Unité dans la diversité » regroupe près de huit cents femmes 25 de toute provenance pour ce moment vécu comme "refondateur». Deux idées se dessinent. Il s'agit d'une part de distinguer dorénavant les espaces subordonnés au FSLN, et les associations de femmes qui entendent différencier travail partisan et engagement pour la cause des femmes. Ces dernières s'auto-dénommeront « mouvement autonome».

Il est question d'autre part de réfléchir au type d'organisation souhaitable pour réunir tous ces collectifs. Les militantes défenseuses de l'autonomie se divisent alors : certaines préfèrent la configuration par réseaux thématiques horizontaux et ouverts, capables de réunir sur tout le territoire à la fois des individus et des collectifs, à la manière de " coalitions d'intérêts ». Sept réseaux sont alors créés en 1992 : alphabétisation, sexualité, économie, environnement, communication, santé et violences. Leurs fondatrices entendent coordonner au plan national les initiatives locales en s'inscrivant dans l'air du temps des transitions démocratiques de l'Amérique latine : défendre les droits des femmes au nom des droits humains et citoyens. Pour ces militantes, le fait de se retrouver autour d'une identité féministe n'est implicitement pas prioritaire, cette question dépendant des positions de chacune. Mais d'autres rejettent avec force cette fragmentation thématique qu'elles jugent facteur de dépolitisation. Elles fondent par conséquent le Comité national féministe

24. Sur le caractère « hybride » des actions collectives et sur le foisonnement des ONG relatives à la cause des femmes en Amérique latine, voir trois travaux de Sonia Alvarez : "Latin American feminisms 'go global': trends of the 1990s and challenges for the new millenium" in Alvarez S., Dagnino E., Escobar A. (eds), Cultures of Politics/Politics of Culture: Revisioning Latin American Social Movements, Boulder, Coo., Westview Press, 1998, pp. 293-324. ; "Advocating Feminism: The Latin American feminist NGO 'boom"”, International Feminist Journal of Politics, 1(2).1999, pp. 181-209; "Beyond NGO-ization? Reflections from Latin America”, Development, 52(2), 2009, pp. 175-184.

25. Encuentro Nacional de Mujeres Por la unidad en la diversidad, 1992, Managua, Centro Editorial de la Mujer, CEM. 
(CNF) privilégiant ainsi la persistance d'un petit groupe de militantes se définissant féministes, et ce afin de préserver et de promouvoir le caractère politique d'un mouvement jugé par elles en situation de fragmentation et de dispersion en raison de son «ONGisation ».

Cette alternative entre front unitaire d'individus féministes et mise en réseau d'ONG généralistes ou spécialisées autour d'un thème d'actions donnera lieu à des débats particulièrement vifs sur l'identité, les formes d'articulation et les projets du «mouvement autonome des femmes » au cours des décennies quatre-vingt-dix et deux mille. Ils se déploieront essentiellement autour de deux organisations : le CNF, et le seul réseau pérenne issu de la rencontre de 1992, le Réseau des femmes contre la violence (RFCV). Bien que la militance féministe nicaraguayenne ne se réduise pas à ces deux groupes ${ }^{26}$, le CNF et le RFCV 27 ont « donné le la » des débats stratégiques des féminismes nicaraguayens, en raison de leur capacité à se constituer en mobilisations à vocation nationale, et à avoir des représentantes dans tout le pays.

Le groupe le plus enclin à questionner le devenir du mouvement féministe post-sandiniste est précisément celui qui a eu pour ambition de le construire. L'objectif des militantes du CNF a toujours été celui-ci, au risque paradoxal de privilégier la radicalité aux dépens du rassemblement large, et d'être perçues comme un réseau d'interconnaissances resserré, d'abord soudé autour d'une érudition théorique féministe peu inclusive et élitiste. Toutes ou presque issues de la génération des sandinistes révolutionnaires des années soixante-dix et quatre-vingt, les militantes du CNF 28 ont affiché leur volonté non seulement de représenter une voix féministe au sein d'instances de dialogues État/société civile, mais aussi de promouvoir leurs thèses auprès d'autres espaces de militance et de provoquer régulièrement des « rencontres fémi-

26. Nous pensons par exemple au «mouvement María Elena Cuadra », un syndicat des travailleuses des maquilas, mais aussi au Réseau des Femmes de Matagalpa, Réseau des Femmes du Nord, ou encore au Comité des Femmes Rurales.

27. Le réseau (qui compte à l'heure actuelle plus de cent collectifs féminins et une vingtaine de militantes à titre individuel), ne dispose pas de personnalité juridique, qui lui est prêtée par une organisation membre. Le RFCV n'a donc pas le statut d'ONG, bien que la plupart de ses participantes soient membres ou salariées d'ONG locales. L'instance décisionnelle ultime est (en théorie) son assemblée générale, les délibérations se font par consensus après débats, et l'espace est ouvert. Chacune peut s'inscrire aux commissions de travail. Seul l'accès au droit de vote pour l'élection des membres de la commission coordinatrice / commission exécutive est soumis à la régularité de la participation en assemblée générale. On peut être membre en tant que représentante de son organisation, ou à titre individuel. Oxfam Angleterre a largement contribué à financer le fonctionnement du RFCV.

28. Une petite cinquantaine de femmes métisses, issues de la classe moyenne, se sont organisées en sections territoriales («capitulos territoriales») dans les principales villes de la Côte pacifique qui délibèrent en assemblée générale à Managua. Elles sont membres du comité à titre individuel et non en représentation d'ONG, et y accèdent par invitation. Le CNF n'a pas de personnalité juridique, mais certaines des activistes sont dirigeantes d'ONG prestataires de services, représentantes d'ONG internationales, consultantes, ou leaders de collectifs locaux. Le CNF a essentiellement financé ses activités de diffusion des thèses féministes avec les fonds de Terre des Hommes-Allemagne. 
nistes » dans la capitale Managua. Selon elles, l'articulation du mouvement doit passer par la reconnaissance des effets fragilisants de «l'ONGisation » qui détourne l'action collective d'objectifs politiques. Elles dénoncent la mise en concurrence des ONG pour l'obtention de fonds qui désolidarise les liens militants. Elles soulignent le déplacement d'un langage des droits vers celui d'une rhétorique gestionnaire : l'ère des manifestes politiques aurait cédé la place à celle des «plans de développement stratégiques». Dans ce contexte, le "projectisme» (proyectismo ou militance professionnalisée de court terme), non dénué de pratiques de corruption, l'emporterait sur une vision de long terme et sur la mise en commun des revendications. Les répertoires d'action se verraient gouvernés par les agendas et les conditions de la coopération au développement. Enfin, la réductibilité des mobilisations féminines à une visée principale d'action serait un obstacle à l'analyse collective des conjonctures politiques et économiques nationales, ce qui est une critique directe au Réseau des femmes contre la violence.

La constance de ces formulations, de ces critiques et de leurs objectifs, contraste fortement avec l'histoire du Réseau des femmes contre la violence, qui donnera à la fois tort et raison aux analyses des militantes du CNF. Aujourd'hui seul mouvement implanté sur tout le territoire rassemblant une diversité d'organisations (près de cent cinquante ONG ou collectifs urbains et ruraux) et fort connu de la population, il est à l'origine d'une avancée légale majeure, la pénalisation des violences intrafamiliales (loi 230 - 1996) et de l'accompagnement d'une action semi publique entièrement financée par la coopération au développement : les Comisarías de la Mujer y de la Niñez, antennes de police spécialisées dans l'accueil aux femmes et enfants victimes de violences intrafamiliales et sexuelles. Construit à mesure de la professionnalisation des militantes et de l'institutionnalisation des ONG prestataires de services qui le composent, le RFCV se consolidera au cours des années quatrevingt-dix et deux mille dans une véritable hybridation entre mouvement articulé capable de dépasser son expertise initiale et mise en réseau « d'entreprises sociales 29 » pour l'exercice des droits des femmes.

29. L'expression est de Murdock D., When Women Have Wings: Feminism and Development in Medellin, Colombia, Ann Arbor, MI, University of Michigan Press, 2008, citée dans Alvarez, S., op. cit., 2009, p.177. Elle fait écho à la sociologie de la mobilisation des ressources selon les termes posés en 1977 par McCarthy et Zald, McCarthy J.D., Zald M. "Resource Mobilization and Social Movements: a Partial Theory”, American Journal of Sociology, vol. 82, 1977, p. 1212-1241. Il s'agit de signifier ici que les organisations fondées par des entrepreneures de cause - ici les leaders d'ONG dont le fil directeur est la lutte contre les violences intrafamiliales et sexuelles - sont l'outil central de construction d'une action collective. Par les financements recherchés et captés, mais aussi par les conditions de leur attribution, ces organisations reposent sur des schèmes rationnels de division du travail, et sont amenées à fonctionner de manière hiérarchique. Dans une perspective similaire, le travail de Matthieu Hély sur la professionnalisation du milieu associatif en France met en avant la notion « d'entreprise associative ». Hély M., Les métamorphoses du monde associatif, Paris, PUF, 2009. 


\section{Se mobiliser contre les violences masculines faites aux femmes avec les ressources de la coopération : un leitmotiv des luttes entre entreprises sociales et mouvement articulé}

\section{Échelles de configuration d'une cause}

L'histoire de la lutte contre les violences masculines à l'encontre des femmes a été, au Nicaragua comme en Amérique latine, marquée par un investissement très important des agendas onusiens et de l'aide publique et privée au développement. Perçue par les institutions publiques comme plus consensuelle que d'autres revendications du féminisme de la deuxième vague telles que l'accès à l'avortement, la publicisation des violences conjugales et sexuelles a été relayée par les gouvernements qui ont bien souvent sous-traité des ONG de femmes dédiées à l'accompagnement des victimes auprès de la police et des tribunaux. La politisation de cette cause a reposé sur plusieurs échelles de mobilisations et a correspondu à des fenêtres d'opportunité tant internationales que régionales et locales.

On a vu qu'au Nicaragua, la construction d'un féminisme affranchi du FSLN s'est inscrite pour une grande part dans la problématisation des violences de genre : les premières expériences en marge du Parti se sont constituées autour des droits reproductifs et sexuels et des violences intrafamiliales. À l'international, les conférences de Vienne (1993) et de Pékin (1995) ont contribué à façonner le paradigme « des droits des femmes comme droits humains », en inscrivant les luttes contre les violences dans les stratégies d'actions pour l'égalité femmes-hommes ${ }^{30}$. Au plan régional, la Convention interaméricaine Belem Do Para, ratifiée par le Nicaragua (1995) a contraint les États de légiférer et de mener des politiques publiques relatives aux violences contre les femmes ${ }^{31}$.

L'État nicaraguayen s'est saisi de cet agenda à la faveur des mobilisations du RFCV ainsi que par des programmes de coopération en particulier des

30. Sur les échelles internationales de la construction de la cause des «violences de genre », voir Keck M., Sikkink K., (eds), Activists Beyond Borders: Advocacy Networks in International Politics, Ithaca, NY, Cornell University Press, 1998 et Joachim J., "Shaping the Human Rights Agenda: the case of violence against women" in Meyer M., Prügl E., (eds), Gender Politics in Global Governance, Oxford, Rowman \& Littlefield Publishers, 1999 pp. 142-160.

31. La Convention interaméricaine pour " prévenir, sanctionner et éradiquer la violence contre la femme » a été adoptée le 9 juin 1994 par l'assemblée générale de l'Organisation des États américains. Elle est entrée en vigueur le 5 mars 1995. Elle comprend tant la définition des violences et de la protection des droits des femmes (Chapitre II) que les devoirs des États signataires (Chapitre III) qui s'engagent à « [condamner] toutes les formes de violence contre la femme et conviennent d'adopter par tous les moyens appropriés et sans délais injustifiés, une politique visant à prévenir, à sanctionner et à éliminer la violence. » Y figurent également les mécanismes interaméricains de protection (chapitre IV) et les dispositions générales de la convention (chapitre V) précisant qu' ' aucune disposition de la présente convention ne sera interprétée comme étant une restriction ou une limitation du droit interne des États parties [...] 》 (art. 13) http://www.cidh.oas.org/Basicos/French/m.femme.htm 
pays scandinaves, des Pays Bas, et de l'Espagne, qui ont intégralement financé la construction d'antennes de police spécialisées. Ces Comisarías de la Mujer $y$ de la Niñez (CMN) ${ }^{32}$ ont été ainsi multipliées, à partir de 1993, dans le cadre des actions publiques mises en œuvre par l'Institut Nicaraguayen de la Femme (Instituto Nicaragüense de la Mujer, INIM). On en compte trentedeux à l'heure actuelle. L'autorité policière y est exclusivement féminine, et est accompagnée d'une psychologue et d'une travailleuse sociale. Sa mission est de recueillir les plaintes des femmes et des enfants victimes de violences sexuelles et intrafamiliales et de leur faciliter l'accès à la justice.

\section{Professionnalisation entre ressources et contraintes internationales négociées}

Dès leur création, nombre d'associations de femmes issues de l'expérience révolutionnaire entendent accompagner les phases d'institutionnalisation des CMN en permettant aux dénonciatrices des violences d'avoir accès à une « attention intégrale » à côté des dispositifs policiers, c'est-à-dire à une sensibilisation sur les causes des violences, à un accès aux soins, à l'information sur les lois en vigueur et des conseils juridiques, et parfois à un hébergement d'urgence. Le programme est aussi une manière de vivre de compétences acquises ou simplement d'obtenir des fonds pour des ONG en construction. D'où la participation, de 1994 à 1998, et de 1998 à 2001 (1 ère phase et "phase de transition ») de vingt-deux « centres alternatifs » de femmes puis, de 2001 à 2004 (2e phase), de près de trente-huit centres. Ces ONG locales, toutes issues de l'expérience révolutionnaire, composées le plus souvent d'un exécutif d'une ou plusieurs leaders, et d'au moins une psychologue et une avocate rémunérées à hauteur de cent à cent vingt dollars par mois ${ }^{33}$, ont d'abord établi un contrat avec l'Institut Nicaraguayen de la Femme (INIM), lequel recevait et administrait les fonds, puis, lors de la seconde phase, avec la police, contrepartie bilatérale du projet ${ }^{34}$.

Dès 1996, le RFCV participe à l'institutionnalisation de cette politique tripartite entre INIM, police et centres de femmes, à la fois en représentation des centres mais aussi comme mouvement national qui veut exercer une influence

32. Les travaux les plus détaillés sur l'histoire des "Comisarías" sont le fait de Nadine Jubb : "Gender, Funding, and the Social Order: Contradictions among the State, the Women's Movement, and Donors regarding the Nicaragua Women's and Children's Police Stations", Prepared for delivery at the 2006 congress of the Canadian Political Science Association, Toronto, June 1-3. 2006.

33. Correspondant approximativement au salaire minimum légal à l'époque.

34. Au cours de la première phase, les coopérants danois, espagnols et néerlandais ont financé la construction des antennes de police (dix entre 1993 et 1998), tandis que la coopération norvégienne a pris en charge le financement des différents services, le budget total s'élevant à 1,5 million de dollars. Au cours de la seconde phase, la coopération espagnole est sortie du projet, orientant ses nouvelles actions vers la consolidation de l'appareil judiciaire. Sont restées les agences de coopération néerlandaise, danoise et norvégienne, en sus de l'agence suédoise, octroyant 3 millions de dollars pour trois ans dont près de la moitié aux trente-huit centres alternatifs à nouveau représentés par la « commission Comisarías» du RFCV. Voir Jubb, N., op. cit. 
sur le programme et négocier avec les coopérants. Les représentantes du RFCV s'assoient régulièrement aux côtés des financeurs, des représentants de la police nationale et de l'INIM.

De très grandes tensions vont opposer les acteurs du projet sur les modes de financement même, et sur le contrôle exercé par l'INIM sur le travail des ONG. Lors de la première phase du projet, les représentantes du RFCV en dénoncent le caractère "néolibéral ", qui place au centre non pas les usagères des services mais les opérations comptables : les centres doivent dresser la liste chaque mois des conseils juridiques ou des accompagnements psychologiques octroyés, et les tarifer - souvent, par souci d'une « efficacité de gestion » en distinguant le coût des types de délit dénoncés, viols ou violences conjugales afin d'espérer être ensuite remboursés dans les meilleurs délais. Les travailleuses-militantes des centres dénoncent le défaut de régulation et d'humanité du programme et estiment que la police ne peut être le seul point d'entrée des usagères. De plus, aucun remboursement des services n'est effectué si les victimes ne passent pas d'abord par la police. Les remboursements sont limités à trois consultations psychologiques par femmes, alors que ces dernières ont bien souvent besoin d'un accompagnement de long terme. Les représentantes des centres font aussi toute une série de constats sur de graves dysfonctionnements policiers et judiciaires, notamment la pratique fréquente des « accords extra-judiciaires » entre victimes et auteurs des délits, contraire aux dispositions légales. Elles remarquent enfin le détournement régulier des matériels à destination des Comisarías par la police "généraliste ", comme les voitures de patrouilles servant aux enquêtes et à l'arrestation des prévenus.

Pendant près d'un an et demi de «phase de transition » (1998-2001) marquée par l'instabilité des financements, les membres du RFCV plancheront sur les modalités d'amélioration de ce programme, en accord avec les bailleurs de fonds. Elles créeront une commission ad hoc et chercheront à centrer les nouvelles modalités d'actions non plus sur l'efficacité de gestion, mais sur les usagères des services désormais considérées comme des survivantes - sobrevivientes - des situations de violence dans une perspective d'empowerment et d'exercice des droits citoyens, mais aussi dans une visée collective de mise en commun des savoirs sur les violences intrafamiliales et sexuelles entre différents acteurs locaux (médecins, juges, policières). Elles y parviendront après de longues négociations avec les coopérants, la police, et l'INIM. À partir de 2001 sera établi un « modèle intégral de prévention, d'attention et de sanction des violences » où les «centres alternatifs » constitués en « réseaux de services » seront aussi considérés comme des points d'entrée pour les femmes en situation de violence. Des «feuilles de références » entre différentes institutions permettront d'assurer le suivi des plaignantes et de mieux collecter les preuves. Des « commissions intersectorielles » locales recevront des financements pour réunir les différents fonctionnaires locaux sous la coordination des promotrices des centres. Les centres ne seront plus remboursés, mais rece- 
vront une attribution mensuelle (entre quatre cents et mille dollars environ) pour couvrir les frais de fonctionnement, louer un local, rémunérer les salariées et gérer les différentes activités prévues dans le programme. En outre, devant l'inefficacité de l'INIM et son instabilité institutionnelle lors de la première phase, c'est la police qui désormais sera la contrepartie officielle des bailleurs, mais la gestion administrative sera menée par un organisme séparé, au sein duquel RFCV, INIM et police siègeront pour en contrôler la mise en œuvre. Enfin, condition imposée par les bailleurs, c'est depuis ce comité que les trois acteurs nicaraguayens parleront d'une seule voix auprès des coopérants. Les représentantes du RFCV, fort réticentes mais contraintes à la négociation avec les fonctionnaires de la police et d'État, obtiennent en contrepartie des financeurs qu'eux aussi ne soient représentés que par un seul interlocuteur.

À l'échelle des centres, la participation au projet rime avec professionnalisation et capacité d'expertise, mais aussi avec bureaucratisation et hiérarchisation entre promotrices leaders et travailleuses rémunérées. La prestation de services et ses conditions de financement signifie aussi devoir rendre compte des activités comptables des ONG, lesquelles sont évaluées par des consultants externes tandis que police et INIM ont implicitement un droit de regard sur le travail mené auprès des survivantes des violences. Entre contraintes et ressources, ces « entreprises sociales » de la lutte contre les violences, sont aussi des espaces à caractère hybride en quête de diversification de financements pour la survie organisationnelle (toujours précaires, à l'image du projet CMN), travail d'amélioration de la mise en œuvre des programmes auxquels elles prennent part, et travail de coordination associative au plan local. À titre d'exemple, l'un de ces centres de la ville de Chinandega, dont la leader charismatique est là encore issue de la branche féminine du syndicat agricole sandiniste, est aujourd'hui à cheval entre son engagement au sein du RFCV, et ses activités locales financées au coup par coup en fonction des programmes dans lesquels elle peut s'inscrire. Au cours des cinq dernières années, elle a obtenu des fonds tantôt pour réunir un groupe de parole sur les violences (Oxfam), tantôt pour rémunérer psychologue et avocate (coopération bilatérale $\mathrm{CMN}$ ), tantôt pour mener une campagne de prévention contre le sida, tantôt pour participer à un programme de l'USAID (agence de coopération des ÉtatsUnis) sur les résolutions extra-judiciaires dans les zones rurales proches. Le temps consacré à toutes ses activités multi-facettes plus ou moins liées à une problématique de genre, relève d'une adaptation stratégique permanente aux ressources proposées par les programmes de développement, tout autant que de la mise en œuvre d'un agenda propre. 


\section{"NGO women's movement model 35 » capacité de rassemblement et montée en généralité féministe}

Toutes les militantes du RFCV ne sont pas parties prenantes de cette action semi-publique. Néanmoins, l'histoire de ce réseau a été profondément marquée par sa participation à l'institutionnalisation des CMN qui a donné à ses représentantes une légitimité et une reconnaissance publique indéniables.

Les tensions sur les difficultés de durabilité de ce programme et sur la consolidation du RFCV sont allées néanmoins de pair, donnant partiellement raison aux voix critiques du CNF. Car, si l'expérience des centres du RFCV n'est qu'un exemple parmi d'autres des modalités « d'ONGisation » de l'action collective des femmes, elle revêt une caractéristique singulière : tandis que certaines $\mathrm{ONG}$ se déploient ou survivent par leur lien direct avec les bailleurs de fonds, les centres qui travaillent en collaboration avec les CMN sont eux soumis à une triple interaction avec la police, l'État, et la coopération bilatérale.

À plusieurs reprises, de nombreux dilemmes sur les statuts organisationnels de l'association se sont posés : faut-il constituer le RFCV en fondation pour mieux représenter et mettre en ouvre le travail de prestations de services et obtenir plus de financements ? S’agit-il plutôt de réunir les centres dans une coalition séparée pour préserver la vocation contestataire du RFCV 36 ? Comment rendre durables les activités des centres entièrement dépendants des bailleurs de fonds, étant donné les atouts que présente aussi leur expertise pour le RFCV ? Plus prosaïquement, comment maintenir tous ces emplois salariés qui, s’ils disparaissaient, détourneraient les militantes de leur " politique du quotidien » à l'adresse des femmes ? Le temps conséquent et bénévole que les militantes du RFCV ont dû consacrer à l'amélioration du programme, celui qu'elles ont dû dédier à la résolution des conflits internes, alors même que toutes ces activités consistaient en une sous-traitance à bas prix d'un rôle imputable à l'État, ont été montrés du doigt par les féministes les plus radicales, notamment celles du CNF.

35. Ewig C., “The Strengths and Limits of the NGO Women's Movement Model: Shaping Nicaragua's democratic institutions", Latin American Research Review, 34(3), 1999, pp. 75102. L’auteure a mené son enquête sur le Réseau des femmes pour la santé, issu de la Rencontre «Unité dans la Diversité » de 1992, dont des dissensions internes ont sonné le glas en 2002. Nous reprenons son expression de "modèle de mouvement de femmes articulé autour d'ONG » (NGO women's movement model) dans la mesure où le Réseau des femmes contre la violence est une action collective structurée aussi autour de liens de «solidarité, de croyances et d'objectifs partagés, afin de mener conjointement des actions protestataires » par-delà la représentation et les intérêts des ONG qui le composent. Nous utilisons ainsi la notion de « mouvement social » dans la mesure où elle fait sens pour les militantes nicaraguayennes et nous nous inspirons de la définition proposée par Della Porta et Diani : Della Porta D., Diani M., Social Movements, an Introduction, Oxford, Blackwell Publishing, 1999, p.16. 
En dépit de cette dépendance à la coopération au développement, le réseau s'est consolidé depuis sa création en 1992, tant en termes de visibilité publique que d'identité collective ou d'organisation interne. Son objectif, la lutte contre « les violences à l'encontre des femmes ", s'est construit et précisé au gré des revendications légales et des investigations menées par ses participantes. Partant d'une appréhension « familialiste » des violences, et de leur reconnaissance comme un problème de santé publique (décret du Ministère de la Santé de 1996), elles ont peu à peu revendiqué une identité féministe et se sont prononcées sur les conjonctures politiques nationales.

En 1998, le scandale provoqué par la dénonciation de Daniel Ortega par sa fille adoptive pour viols alors qu'elle était enfant a catalysé la rupture du mouvement avec les instances et avec une certaine mythologie historique du parti sandiniste ${ }^{37}$. Ce soutien officiel à Zoilamérica Narváez lors de l'affaire publique qui l'a opposée au « héros révolutionnaire » Ortega, mais aussi la participation active à des controverses complexes autour de « cas emblématiques » - l'affaire Rosita ${ }^{38}$ de 2003 par exemple - ont projeté le RFCV comme un mouvement de résistance au patriarcat, et de promotion de la démocratie comme condition de lutte contre l'impunité. Surtout, c'est à l'aune de ces controverses publiques que les membres du RFCV ont entamé en interne des débats sur l'avortement, sur les violences sexuelles, sur la laïcité, sur l'articulation du pouvoir politique et de l'ordre sexué, choses longtemps non débattues. À plusieurs reprises, les militantes ont participé à des marches anti-corruption. Ainsi, alors que le RFCV était à ses débuts un petit groupe de femmes censées mener des actions de lobbying sur les violences intrafamiliales sous la forme d'un réseau thématique, il est devenu, par son approfondissement de la définition même des violences masculines et par la professionnalisation de ses militantes, un mouvement à très forte capacité de mobilisation, sociologiquement proche des «mouvements populaires » de femmes, et dont la construction empirique de ses lectures féministes l'ont amené à dépasser largement son expertise et sa revendication initiales.

Ainsi, les critiques sur le détournement des objectifs du mouvement féministe sous l'effet de «l'ONGisation » et des « agendas imposés » de la coopération internationale méritent d'être nuancées au vu de l'histoire du RFCV.

36. C'est le cas depuis 2004 : les centres se sont réunis en «Alliance des centres » pour éviter toute confusion entre RFCV et politique de prestation de services. Les centres continuent néanmoins de participer au RFCV.

37. Lacombe D., "L'affaire Zoilamérica Narváez contre Daniel Ortega ou la caducité de "l'homme nouveau" ", Problèmes d'Amérique Latine, n 73, été 2009, pp. 73-100.

38. Le RFCV (et d'autres mouvements de la société civile) a pris la défense de cette petite fille de neuf ans victime d'une grossesse forcée suite à des viols répétés de son père adoptif. Alors que l'État lui déniait le droit d'avorter, en bafouant la législation en vigueur, les membres du réseau alliés à d'autres représentants d'ONG et de mouvements ont permis à Rosita l'interruption de grossesse. 


\title{
Débats sur les partenariats internationaux en contrepoint des enjeux politiques nationaux
}

\author{
"Mobilisation sans émancipation 39 ? "
}

Cela étant, les capacités de mobilisation et de consolidation d'une coalition d'ONG, sous l'effet croisé de la professionnalisation et de la montée en généralité féministe à partir d'un « entreprenariat » sur les violences de genre, n'ont pas épuisé les débats internes à la militance. À la manière là encore d'un déplacement d'une réflexion déjà formulée dans les années quatre-vingt, certaines féministes issues de la deuxième mouvance étudiée ici, le Comité national féministe, n'ont eu de cesse de décrier la fragilité et la précarité d'une mobilisation sans quête, selon elles, d'une "véritable émancipation " des femmes, devant passer par la mise en mouvement d'un «sujet politique » capable de s'engager pour la démocratisation du pays.

Rappelons la définition que les militantes donnent à leur groupe : « Le CNF surgit comme un espace de résistance face à la dépolitisation et la désidéologisation du mouvement, et pose en impératif la nécessaire articulation de ce dernier comme un sujet politique. Il s'agit d'une proposition plus organique et structurée, qui entend s'articuler depuis les bases territoriales et la conviction individuelle de ses membres. Il cherche à renouer avec le discours radical et démocratique qui a caractérisé la lutte féministe, ainsi qu'avec son caractère contestataire face à l'autoritarisme de l'État et de la société 40 ».

En effet, depuis sa création, les militantes du CNF ont voulu construire en substance un mouvement féministe à la mesure des obstacles politiques et sociaux de l'histoire politique nicaraguayenne. Forcées de reconnaître qu' « ONGisation » et professionnalisation avaient contribué à façonner la militance, elles ont continué d'avancer que la cause féministe méritait d'être approfondie et repolitisée pour mener des actions autres que celles consistant à mettre en œuvre des programmes de la coopération internationale. Elles faisaient un triple constat. Premièrement, en dehors des avancées relatives aux luttes contre les violences intrafamiliales et sexuelles, les gouvernements nicaraguayens successifs avaient fait obstacle aux revendications féministes. Deuxièmement, la fermeture progressive du jeu politique et les accointances Églises-partis-institutions publiques ${ }^{41}$, dès après le mandat de Violeta

39. Nous empruntons là aussi l'expression, reprise par les féministes nicaraguayennes pour analyser le destin de leur action collective au cours du régime sandiniste, à Molyneux M., op. cit.

40. In Feminismo y globalización: Apuntes para un análisis político desde el Movimiento, [Convención Feminista, volver al escándalo y la transgresión, Por una agenda propia y autónoma] Comité Nacional Feminista, 2003, p.50.

41. Toute la période de redéploiement de l'action collective féminine post-sandiniste a coincidé avec deux mandats présidentiels très nettement marqués par la réactivation des pratiques caudillistes au sein de l'exécutif comme au sein du parlement. L'accès au pouvoir d'Arnoldo Alemán, en 1997, a été synonyme d'un approfondissement de la corruption et du clientélisme, 
Chamorro (1990-1996), empêchaient l'accès à tout canal de participation susceptible de faire valoir les revendications féministes et de modifier substantiellement les politiques de genre des gouvernements ${ }^{42}$. Enfin, elles pointaient les risques d'un retour au pouvoir de leurs alliés d'armes d'antan, le FSLN, contre lesquels elles avaient largement défini et redéployé leur féminisme et qui devenait pour elles un « ennemi principal ». Certaines d'entre elles proposaient alors de donner forme à une nouvelle mise en mouvement féministe, capable de s'allier avec des partis politiques aux aspirations démocratiques, et en faisaient un objectif premier. Elles fondaient en 2006 le « Mouvement autonome des femmes » (MAM), un mouvement resserré autour d'une centaine de leaders de différentes provenances engagées à titre individuel et non en représentation d'ONG et pratiquant une politique contestataire contre le pouvoir reconduit du FSLN à la présidence et au parlement (2006-2010). D'autres, opposées à cette stratégie faisant le jeu (considéré comme vain) de la politique conventionnelle, créaient le "mouvement féministe ", toujours à vocation autonome, mais aux visées sensiblement différentes : rassembler le plus de femmes et d'associations possibles (une dizaine d'associations et de nombreuses activistes), et à défaut de pouvoir infléchir les configurations électorales et partisanes, continuer de mener, avec les ressources de la coopération internationale, une diffusion des thèses féministes sous la forme d'ateliers et d'actions protestataires lors de manifestations publiques.

\section{Entre adaptabilités stratégiques et politiques de résistance féministe}

Au fond, dans ce cadre, l'appel à sortir d'un quant à soi des ONG pour penser les conditions d'articulation des actions collectives a été constant. De la même manière, la préservation des $O N G$ et leur survivance a été un objectif en soi. À l'heure où les financements de la coopération se tarissent, la durabi-

les convictions personnelles du président et ses alliances avec l'Église catholique ont pris radicalement le pas sur la (pourtant) constitutionnelle laïcité. À la même époque, le pacte parlementaire signé en 1999 entre Daniel Ortega (FSLN) et Arnoldo Alemán (Parti libéral constitutionnaliste) à des fins économiques, politiques et judiciaires, a sonné le glas d'un jeu des concurrents pour le pouvoir libre et pluraliste à peine esquissé sous Chamorro, au profit de la main mise des partis sur les institutions de contrôle de l'État. L'accord politique a notamment consisté à attribuer des sièges en fonction des représentations partisanes du PLC et du FSLN, au sein de la Cour Suprême de Justice, du Conseil Suprême Electoral. Il a servi également à protéger Alemán et Ortega des poursuites judiciaires, l'un pour corruption, l'autre pour viol sur sa fille adoptive. Le prolongement de cet «accord de caballeros» au parlement, sous la présidence d'Enrique Bolaños (2001-2006) a provoqué une crise institutionnelle et politique majeure au cours de ce mandat présidentiel, paralysant le pouvoir exécutif par le pacte parlementaire.

42. À titre d'exemple, sous la présidence d'Arnoldo Alemán, a été institué « el día del no nacido » - « journée de l'enfant à naître ", et ont été promues à plusieurs reprises des marches publiques contre l'avortement, y compris thérapeutique. L'Institut Nicaraguayen de la Femme a manqué de peu d'être rattaché au ministère de la famille au nom d'une vision traditionaliste des rôles masculins et féminins. Arnoldo Alemán a également orchestré des poursuites judiciaires et une pression fiscale à l'égard d'ONG de femmes au motif qu'elles pratiquaient des avortements. Voir Kampwirth K., “Arnoldo Alemán takes on the NGO's: Antifeminism and the new populism in Nicaragua", Latin American Politics and Society, summer 2003. 
lité et l'autosuffisance d'un maillage associatif qui vit majoritairement par des projets de court-terme pose un véritable problème, non seulement pour les organisations professionnalisées mais aussi dans la perspective d'une mise en mouvement des femmes. En outre, l'histoire de la survivance des ONG est aussi une affaire politique : à la fin de l'année 2008, le pouvoir exécutif et les médias inféodés au FSLN ont mené une campagne de persécution publique à l'égard d'associations et ONG critiques du pouvoir, parmi lesquelles le «mouvement autonome des femmes », le "grupo Venancia », le «Centre d'investigation sur la communication ", au motif qu'elles défendaient l'avortement, lequel était pénalisé sous toutes ses formes deux ans auparavant. Cette tentative d'intimidation et de contrôle de la société civile (déjà expérimentée sous le mandat d'A. Alemán - 1997-2001), a aussi concerné les ONG internationales Forum Syd et Oxfam Grande Bretagne, qui ont dû rendre compte de leurs activités auprès du ministère public. Avec les mêmes intentions, sept activistes du Réseau des femmes contre la violence et du Mouvement autonome des femmes (MAM) ont été accusées en 2007, par un groupe catholique, d'association de malfaiteurs et d'apologie de l'avortement. Les charges ont finalement été levées en 2009 par le ministère public, après une pression constante exercée sur ces militantes.

Dans ce contexte, les analyses portées sur les formes de globalisation du genre et des partenariats internationaux sont intervenues en contrepoint des enjeux politiques nationaux. Les militantes ont tantôt négocié avec ces partenaires pour constituer des alliances, tantôt publiquement fustigé leur prétention implicite de neutralité devant des décisions publiques anti-féministes. Elles ont critiqué l'incapacité des partenaires internationaux à insérer les programmes de développement dans la société civile, tourné en dérision leur ignorance des enjeux politiques internes ou encore dénoncé leur arrogance néocolonialiste. Mais la tendance générale de cette militance féministe n’a pas été de poser la question de l'autonomie dans les termes mentionnés plus haut, c'est-à-dire à la façon d'un courant libertaire rejetant les espaces d'action et de négociation internationaux ou nationaux et dénonçant l'institutionnalisation de l'action collective féministe. Rarement dupes du caractère néolibéral de l'industrie du développement, les militantes ont défini leur autonomie par la priorisation d'une rupture avec les allégeances partisanes et par des stratégies de contournement ou de captation opportune et sélective des ressources présentées par l'aide au développement ${ }^{43}$. Et la tendance à leur institutionnalisation a aussi été synonyme de subversion féministe. L'une des hypothèses explicatives de ces dynamiques est celle d'une génération de militantes dont les habitus sont encore empreints d'un mouvement révolutionnaire pour lequel le changement social et les perspectives utopistes passaient par la prise

43. Certaines d'elles se sont par ailleurs implicitement saisies d'approches critiques du courant autonome latino-américain pour instiller au féminisme issu de la révolution une idéologie plus radicale contre le racisme et l'hétéro-normativité, choses longtemps délaissées ou tabous dans de nombreux espaces. 
de pouvoir ou la pratique de conscientisation des masses. Mais cet essai de construction de marges de manœuvre, même réduites, entre contraintes et ressources internationales et obstacles à l'échelle nationale, relève de pratiques dont la militance nicaraguayenne ne constitue pas l'exception ${ }^{44}$. Elles invitent à explorer, par-delà la polarisation entre autonomes et institutionnelles (qui continue de résonner et de structurer les débats en Amérique latine), la diversité des formes d'actions et le sens que les militantes leur accordent dans leur construction de la cause des femmes.

44. Dans une tout autre configuration et histoire du féminisme voir les analyses de Sophie Stoffel, op. cit. 